Article

\title{
Superhydrophobic/Flame Retardant/EMI Shielding Fabrics: Manufacturing Techniques and Property Evaluations
}

\author{
Hao-Kai Peng ${ }^{1,2,3}$, Yanting Wang ${ }^{1}$, Ting-Ting $\mathrm{Li}^{1,2}{ }^{1}$, Ching-Wen Lou ${ }^{1,3,4,5,6,7, * \mathbb{C}, \mathrm{Qi} \mathrm{He}}{ }^{1}$ and \\ Jia-Horng Lin $3,4,6,8,9, *$ (D) \\ 1 Innovation Platform of Intelligent and Energy-Saving Textiles, School of Textile Science and Engineering, \\ Tianjin Polytechnic University, Tianjin 300387, China; skyphk@163.com (H.-K.P.); \\ 18722122269@163.com (Y.W.); litingting_85@163.com (T.-T.L.); h18622343633@163.com (Q.H.) \\ 2 Tianjin and Education Ministry Key Laboratory of Advanced Textile Composite Materials, \\ Tianjin Polytechnic University, Tianjin 300387, China \\ 3 Fujian Key Laboratory of Novel Functional Textile Fibers and Materials, Minjiang University, \\ Fuzhou 350108, China \\ 4 Department of Chemical Engineering and Materials, Ocean College, Minjiang University, \\ Fuzhou 350108, China \\ 5 Department of Bioinformatics and Medical Engineering, Asia University, Taichung 41354, Taiwan \\ 6 College of Texitle and Clothing, Qingdao University, Shangdong 266071, China \\ 7 Department of Medical Research, China Medical University Hospital, China Medical University, \\ Taichung 40402, Taiwan \\ 8 Laboratory of Fiber Application and Manufacturing, Department of Fiber and Composite Materials, \\ Feng Chia University, Taichung City 40724, Taiwan \\ 9 Department of Fashion Design, Asia University, Taichung 41354, Taiwan \\ * Correspondence: cwlou@asia.edu.tw (C.-W.L.); jhlin@fcu.edu.tw (J.-H.L.); \\ Tel.: +886-4-2451-7250 (ext. 3405) (J.-H.L.); Fax: +886-4-24510871 (J.-H.L.)
}

Received: 27 March 2019; Accepted: 27 April 2019; Published: 9 May 2019

\begin{abstract}
Electromagnetic pollution interferes with electronic equipment in proximity and jeopardizes human health, which urges the development of electromagnetic interference (EMI) shielding materials. It is urgent to develop electromagnetic interference (EMI) shielding materials. However, the preparation of materials with superhydrophobicity, flame retardancy and EMI shielding properties is still challenging. In this study, we invented a core-spun yarn feeding device, which uses polysulfonamide (PSA) roving as a coating material and stainless steel wire as the core material to prepare a conductive core-spun yarn, which solves the problem of the wire having an easily exposed fabric surface. The finally prepared conductive fabric was subjected to Waterproof $2 \mathrm{P}$ hydrophobic treatment to form a superhydrophobic flame-retardant EMI shielding fabric. The results show that the hydrophobic treatment creates a thin film over the woven fabrics, and the contact angle of the fabric surface can reach $155^{\circ}$. The hydrophobic treatment will not damage the shielding effect and slightly increase the $\mathrm{dB}$ value. The average $\mathrm{dB}$ value of PSA-SS-1' and PSA-SS-2' are increased by $0.82 \mathrm{~dB}$ and $1.92 \mathrm{~dB}$, respectively. When composed of conductive wrapped yarns for both the warp and weft yarns, the electromagnetic interference shielding effectiveness (EMI SE) of conductive fabrics is beyond $30 \mathrm{~dB}$ at $0-3000 \mathrm{MHz}$ and the burnt depth is shorter than $40 \mathrm{~mm}$. As for real applications, superhydrophobic/flame retardant/EMI SE fabrics can be used in a moist and complex environment with retaining conductivity and shielding effectiveness.
\end{abstract}

Keywords: superhydrophobic; flame retardant; electromagnetic interference shielding; conductive fabric 


\section{Introduction}

Mobile communications and electronic devices have become necessities for people to acquire new knowledge and communicate with others in the modern world [1,2]. The use of electronic products such as laptops and cellphones inevitably generates electromagnetic interference (EMI) [3-5]. The electromagnetic pollution jeopardizes people's health, which leads to the presence of the EMI shielding materials [6,7]. EMI shielding materials attenuate electromagnetic energy propagation by means of absorption and reflection, thereby refraining electromagnetic interference and pollution. The shielding materials are qualified when they have a resistivity lower than $10^{2} \Omega / \mathrm{cm}^{2}[8,9]$. One simple method of shielding EMI is adopting the conductivity of metallic materials. However, the applications of metallic materials are restricted due to having a heavy weight, a high cost, and the difficulty of the process $[10,11]$ and conductive polymer composites (CPC) have been developed in order to address the issue $[12,13]$. Nazan et al. studied the EMI SE of cotton/metallic composite fabrics and found that the plain weave fabrics had an optimal shielding effectiveness [14]. Lou et al. investigated $\mathrm{Cu}$ and SS conductive fabrics, and the differences in the lamination layer and lamination angle could change the electromagnetic interference shielding effectiveness (EMI SE) of materials [15]. Chen et al. compared the methods for gaining EMI SE and subsequently composed a complete shielding network with improved EMI SE [16]. The comfort of conductive fabrics compromises when the constituent metallic wires could not be enwrapped completely [17]. As a result, the conductive fabrics demonstrate oxidation corrosion, which decreases the shielding effectiveness against electromagnetic waves.

Another issue is the stability of the electrical conductivity and, hence, the EMI shielding efficiency and reliability for the practice application of the CPC. In order to better solve the problem of electromagnetic radiation, it is also necessary to maintain the stability of the conductivity of the conductive material. Therefore, the shielding efficiency of the conductive material will be reliable and stable in practical applications [18-20]. For example, when conductive metal materials are used under harsh conditions such as humidity, acidity and alkalinity, the materials are easy to be oxidized or corroded, especially when the composite yarn cannot wrap the wire well [21-23].

Therefore, the conductive material is preferably corrosion resistant. In fact, a fabric having superhydrophobicity is a good candidate for achieving this goal because the superhydrophobic material can repel water droplets, thereby preventing the aqueous solution from diffusing within the conductive fabric $[24,25]$. In addition to maintaining the stability of conductivity during use, the material should also have a certain flame retardant effect to prevent fires caused by electronic equipment.

PSA fiber with good acid and alkali resistance has relatively better combustion resistance, heat resistant property, heat stability, and a limiting oxygen index (LOI) over 33\%. However, PSA fibers have a moisture regain of $5.5 \%$, which has a negative influence on the hydrophobicity of the resulting conductive fabrics in a moist condition [26-29]. In order to develop conductive fabrics with excellent flame retardancy, excellent hydrophobicity and excellent EMI shielding properties, our laboratory has invented a feeding device for preparing conductive core-spun yarns, which is compared to conventional core-spun yarn devices. A custom-made feeding device is used to produce conductive wrapped yarns, ensuring that one or two metallic wires (i.e., the core) can be precisely wrapped in PSA roving to prevent the exposure of metallic wires. The conductive wrapped yarns are then made into conductive fabrics that are then processed with waterproof $2 \mathrm{P}$ superhydrophobic treatment, to prepare a superhydrophobic flame-retardant electromagnetic shielding fabric. After hydrophobic treatment, the contact angles of fabrics reached $155^{\circ}$ and the burnt depth is shorter than $40 \mathrm{~mm}$. The hydrophobic treatment will not damage the shielding effect for which the EMI SE of PSA-SS-1' ${ }^{\prime}$ and PSA-SS-2' are $34.08 \mathrm{~dB}$ and $34.42 \mathrm{~dB}$ with electromagnetic waves of $0-3000 \mathrm{MHz}$. 


\section{Experimental}

\subsection{Materials}

316L Stainless steel wires (SS) (Yuanneng Co., Ltd., Shanghai, China) have a diameter of 0.06 $\mathrm{mm}$, breaking strength of $354.36 \mathrm{cN}$, and an elongation of $29.4 \%$. PSA fibers (Shanghai T \& L Co., Ltd., Shanghai, China) have a fiber length of $51 \mathrm{~mm}$, a fineness of 1.5D, a specification of 21S/3, a tensile strength of $207.84 \mathrm{cN}$ and a tensile elongation of $8.9 \%$. Waterproof 2P (Hefei Dong Fang Mei Jie Molecular Material Technology Co., Ltd., Anhui, China) has a concentration of $2 \%$. Waterproof $2 \mathrm{P}$ is composed of a fluorine emulsion polymer, emulsion fluid, and a weak cationic type that is non-toxic biodegradable.

\subsection{Preparation of Conductive Wrapped Yarns}

A core yarn feeding device, which is specially made with a spinning frame as a drafting system, can fully enwrap the SS wires in PSA roving in the spinning process in order to prevent the exposure and oxidation of metallic wires (Figure 1). The number of metallic wires is adjusted to form core yarns with different functions. The PSA roving with a specification of $4 \mathrm{~g} / 10 \mathrm{~m}$ is used as the sheath while one or two metallic wires are used as the core. The conductive wrapped yarns are made with twist counts of 50,60, 80, 100, and 120 turns $/ 10 \mathrm{~cm}$.

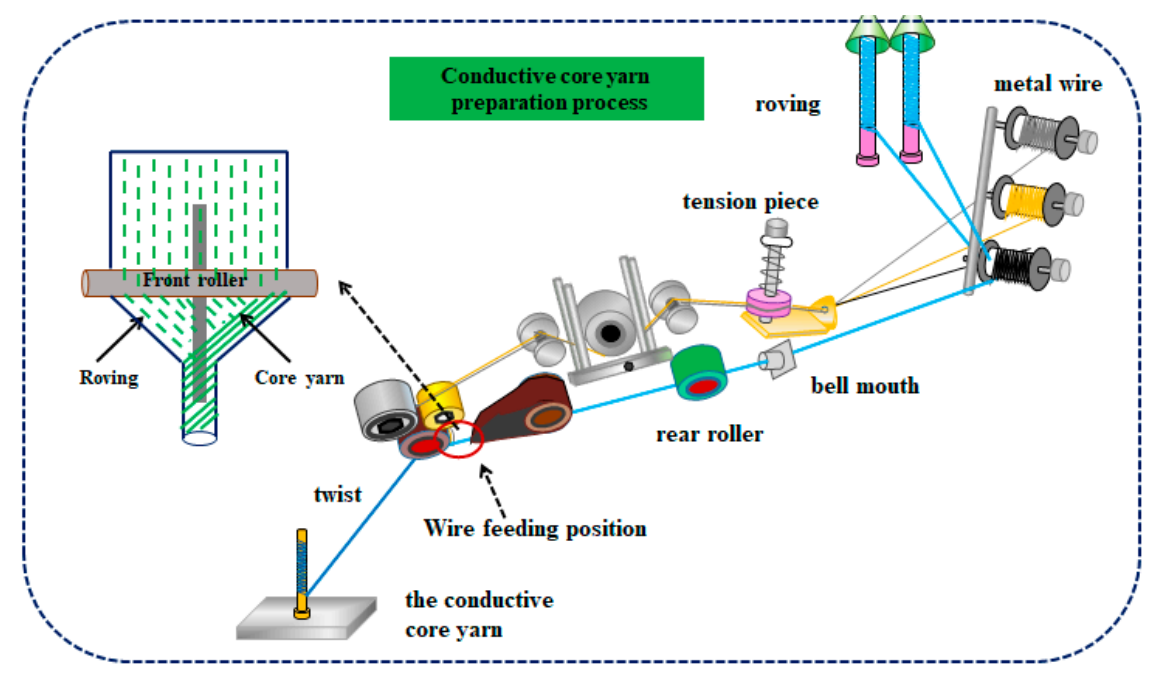

Figure 1. The schematic diagram of the preparation process for conductive wrapped yarn.

\subsection{Preparation of EMI Shielding Fabrics}

A semi-automatic weaving machine (DWL 5016, Hangzhou Tianma Textile Machinery, Zhejiang, China) is used to produce conductive woven fabrics. The PSA yarn and conductive yarn are used as warp or weft. The four fabric specifications are shown in the Table 1 (PSA-SS-1: for warp and weft yarn is a conductive yarn wrapped in a single wire; PSA-SS-W1 indicates that the warp yarn is PSA yarn and the weft is a conductive yarn wrapped with a wire; PSA/SS-60 means feeding one wire with a twist of 60 turns $/ 10 \mathrm{~cm}$; PSA/SS-60' means feeding 2 wires, with a twist of 60 turns $/ 10 \mathrm{~cm}$, and so on).

Table 1. The characteristics of conductive woven fabrics.

\begin{tabular}{|c|c|c|c|c|c|c|}
\hline Fabric Code & Warp Yarn & Weft Yarn & $\begin{array}{l}\text { Fabric Weight } \\
\left(\mathrm{g} / \mathrm{m}^{2}\right)\end{array}$ & $\begin{array}{c}\text { Thickness } \\
\text { (mm) }\end{array}$ & $\begin{array}{l}\text { Number of Warp } \\
\text { Yarns (ends/10 cm) }\end{array}$ & $\begin{array}{c}\text { Number of Weft } \\
\text { Yarns (picks/10 cm) }\end{array}$ \\
\hline PSA-SS-1 & PSA/SS-60 & PSA/SS-60 & 228.74 & 0.41 & 120 & 110 \\
\hline PSA-SS-2 & PSA/SS-60' & PSA/SS-60' & 233.64 & 0.41 & 120 & 110 \\
\hline PSA-SS-W1 & PSA & PSA/SS-60 & 210.92 & 0.38 & 120 & 110 \\
\hline PSA-SS-W2 & PSA & PSA/SS-60' & 214.36 & 0.38 & 120 & 110 \\
\hline
\end{tabular}




\subsection{Preparation of Superhydrophobic/Flame Retardant/EMI Shielding Fabrics}

The conductive fabrics are immersed in waterproof $2 \mathrm{P}$ ( $2 \%$ o.w.f.) and compressed with a pick-up rate of $60 \%$, which is repeated for another cycle. Samples are then dried at $80{ }^{\circ} \mathrm{C}$ for $5 \mathrm{~min}$ and then stored at room temperature. Figure 2 shows the resulted superhydrophobic/flame retardant/EMI shielding fabrics.

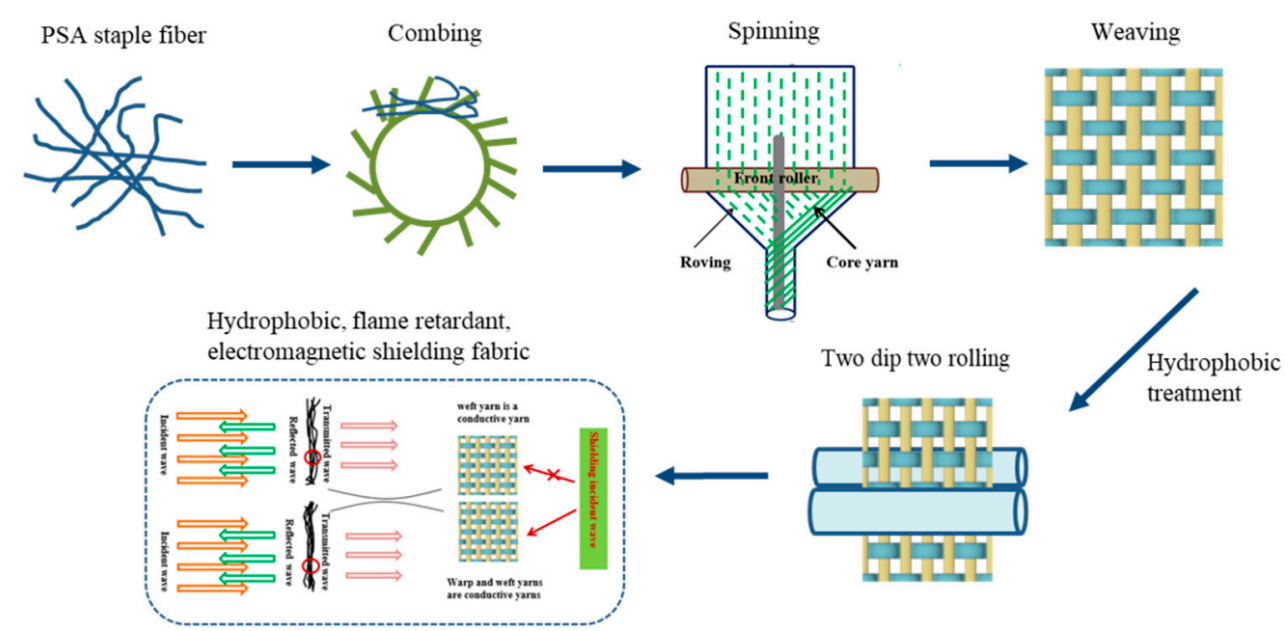

Figure 2. The schematic diagram of the processing procedures of hydrophobic/flame retardant/ electromagnetic interference (EMI) shielding fabrics.

\subsection{Characterizations}

The microstructure of the superhydrophobic and conductive composite fabric was investigated by using a scanning electron microscope (TM3030, HTTACHJ, Tokyo, Japan) at an accelerating voltage of 5 $\mathrm{kV}$. The contact angles (CAs) of the composite fabric were measured by using an optical CA measuring device (OCA20) and a $5 \mu \mathrm{L}$ water droplet was chosen for each CA measurement. The thermogravimetric measurement was conducted with a thermogravimetric analyzer (TG 209F3, NETZSCH, Bavaria, Germany) with dry nitrogen gas at a flow rate of $60 \mathrm{~mL} \cdot \mathrm{min}^{-1}$. The relative mass loss of the samples was recorded from $50{ }^{\circ} \mathrm{C}$ to $800{ }^{\circ} \mathrm{C}$ at a heating rate of $10{ }^{\circ} \mathrm{C} \cdot \mathrm{min}^{-1}$. The thermal properties were analyzed using a differential scanning calorimeter (NETZSCH DSC200F3, Bavaria, Germany). Samples were heated from room temperature to $500{ }^{\circ} \mathrm{C}$ at $30^{\circ} \mathrm{C} / \mathrm{min}$ and held for $3 \mathrm{~min}$ to remove the thermal history. Subsequently, the samples were cooled to $30^{\circ} \mathrm{C}$ at a cooling rate of $10{ }^{\circ} \mathrm{C} / \mathrm{min}$ and heated again to $500{ }^{\circ} \mathrm{C}$ at $10^{\circ} \mathrm{C} / \mathrm{min}$. The tensile properties of the core yarns were measured using a universal force meter (HT-9101, Hong da Instrument, Shanghai, China) with the distance between the gauges being $250 \mathrm{~mm}$ and the tensile rate being $300 \mathrm{~mm} / \mathrm{min}$. EMI shielding performance of the composite fabrics was evaluated by using a spectrum analyzer (Advantest R3132, Burgeon Instrument, Taiwan). All samples were sliced into small circular plates with a diameter of $150 \mathrm{~mm}$ for the EMI shielding tests.

\section{Results and Discussion}

Figure $3 a, b$ shows the cutting section of the conductive wrapped yarns where the metallic wires are confirmed to be wrapped in PSA roving regardless of the number of metallic wires. The observation indicates that the employment of the custom-made feeding device produces wrapped yarns with an intact morphology and thus solves the corrosion problem caused by the exposure of metallic wires. Figure $3 c$ shows the morphology of the control group (PSA-SS-W1) that is not processed with waterproof $2 \mathrm{P}$, whereas Figure $3 \mathrm{~d}$ shows the morphology of the experimental group (i.e., PSA-SS-W1') that undergoes superhydrophobic treatment using waterproof $2 \mathrm{P}$, and the fabric is covered with a transparent breathable film. The major component of the hydrophobic agent is fluorine-containing an emulsion polymer. Polysiloxane is aligned along the same direction with hydrophobic agent-CH3 
being aligned outwards. Moreover, waterproof $2 \mathrm{P}$ is composed of silicon and oxygen atoms that interact with some atoms of the fibers to form coordination bonds and hydrogen bonds. Subsequently, the vapor and air can penetrate the fabrics instead of the water droplets. The fabrics demonstrate a good hydrophobic effect with CAs of $155^{\circ}$. The microstructure of the core-spun yarns after FR testing is shown in Figure $3 \mathrm{e}-\mathrm{h}$. It can be showed that the fabrics do not demonstrate distinct shrinkage, cracking, and dripping during the combustion, after which the fabrics exhibit carbonization and have a crisp edge, retaining good fabric morphology. A film is formed on the surface of the PSA-SS-W1 fabric after hydrophobic treatment. The outermost film will destroy the flame retardant effect of the PSA-SS-W1 fabric. This is also the damaged length of the PSA-SS-W1 sample, which is higher than that of PSA-SS$\mathrm{W} 1^{\prime}$. Figure $3 \mathrm{i}$ shows that water droplets with different $\mathrm{pH}$ values displayed on the surface of the superhydrophobic fabric, including acid and alkali water droplets that can stand well on the fabric surface. This phenomenon shows that the superhydrophobic fabric can be used in harsh conditions such as humid, acid and alkali environments. The thermal properties of fabrics were investigated by PSA-SS-W1 and PSA-SS-W1'. The results show that the hydrophobic treatment will not destroy the stability of the thermal properties. A more detailed discussion is shown in thermogravimetric analysis.
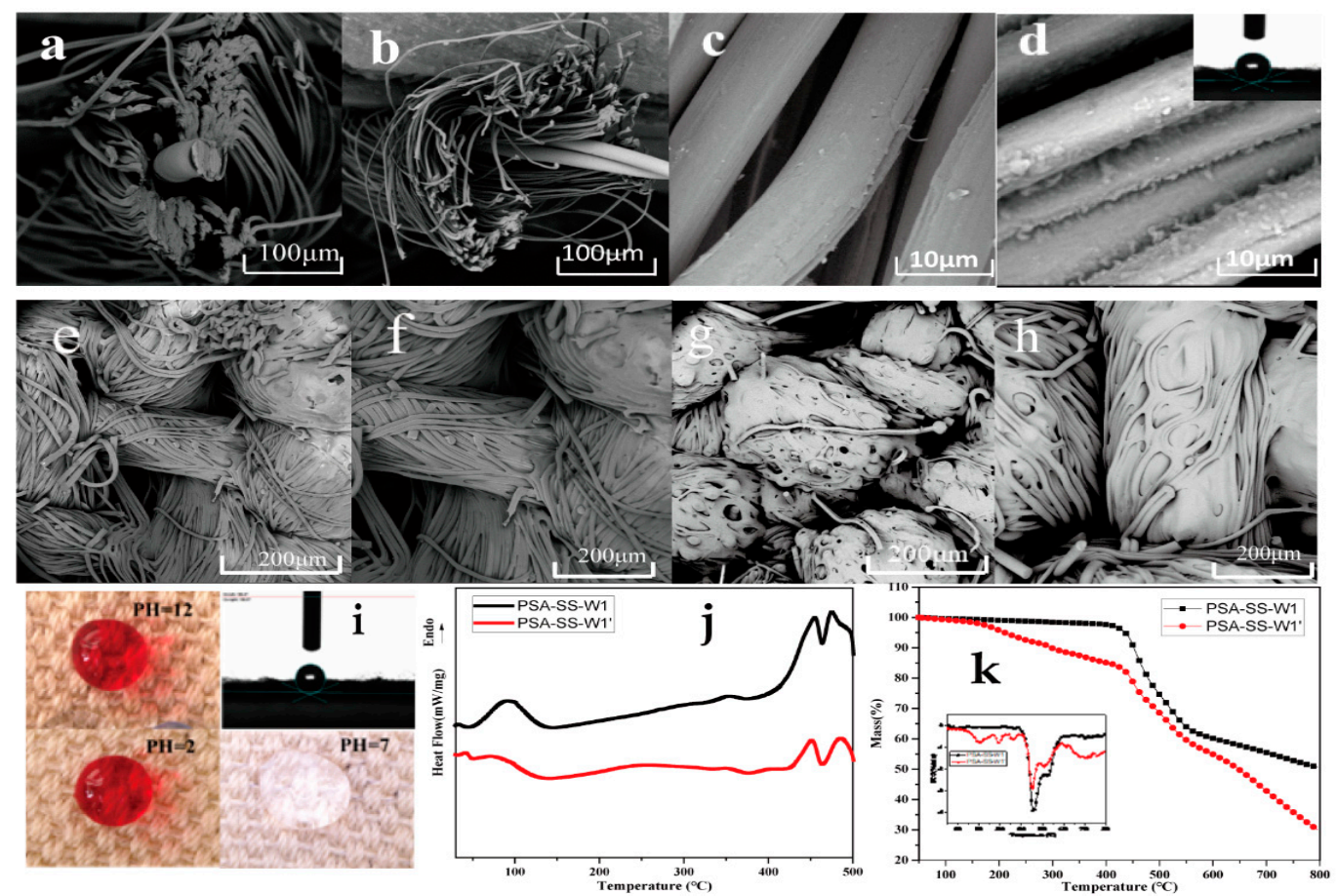

Figure 3. (a,b) The scanning electron microscope (SEM) image of Core yarn with different number of wires; (c) SEM image of the warp yarn is PSA yarn and the weft is a conductive yarn wrapped with a wire (PSA-SS-W1); (d) SEM image of PSA-SS-W1'; (e,f) SEM image after burning of PSA-SS-W1'conductive fabric (g,h): SEM image after burning of PSA-SS-W1 conductive fabric; (i) Photograph displaying water droplets with different $\mathrm{pH}$ values on the superhydrophobic fabric surface; $(\mathbf{j}, \mathbf{k})$ Thermal properties of fabrics before and after superhydrophobic treatment.

\subsection{Breaking Strength of Conductive Wrapped Yarns}

Figure 4 shows the breaking strength of conductive wrapped yarns as related to the twist counts. The breaking strength is correlated with the level of twisting. Increasing the twist counts first increases and then decreases the breaking strength. As for the wrapped yarns composed of one metallic wire, the optimal twist counts is 60 turns $/ 10 \mathrm{~cm}$ with a maximum breaking strength of $1.67 \mathrm{cN} / \mathrm{dtex}$. As for the wrapped yarns composed of two metallic wires, the optimal twist counts is 60 turns $/ 10 \mathrm{~cm}$ with a maximum breaking strength of $1.02 \mathrm{cN} / \mathrm{dtex}$. A low twist count renders the wrapped yarns with uneven strength lengthwise, which generates a greater number of rounds for weak ring arrangement. 
Hence, the wrapped yarns have a higher strength where the weak rings are situated rather than other parts. An increase in the twist counts causes the fibers to incline and decreases the axial force of the core yarns. Therefore, the wrapped yarns demonstrate a lower strength. In addition, the twisting process exerts prestress onto the fibers, attenuating the ability of fibers to withstand a force. To sum up, the core and the sheath of the wrapped yarns jointly form a compact structure and contribute to a greater cohesion when the twist counts increase. Meanwhile, the amount of PSA roving per unit length also increases, which improves the strength and elongation of the wrapped yarns. Conversely, excessive twist counts have a negative influence on the axial force of the wrapped yarns which, in turn, interferes with the mechanical properties.
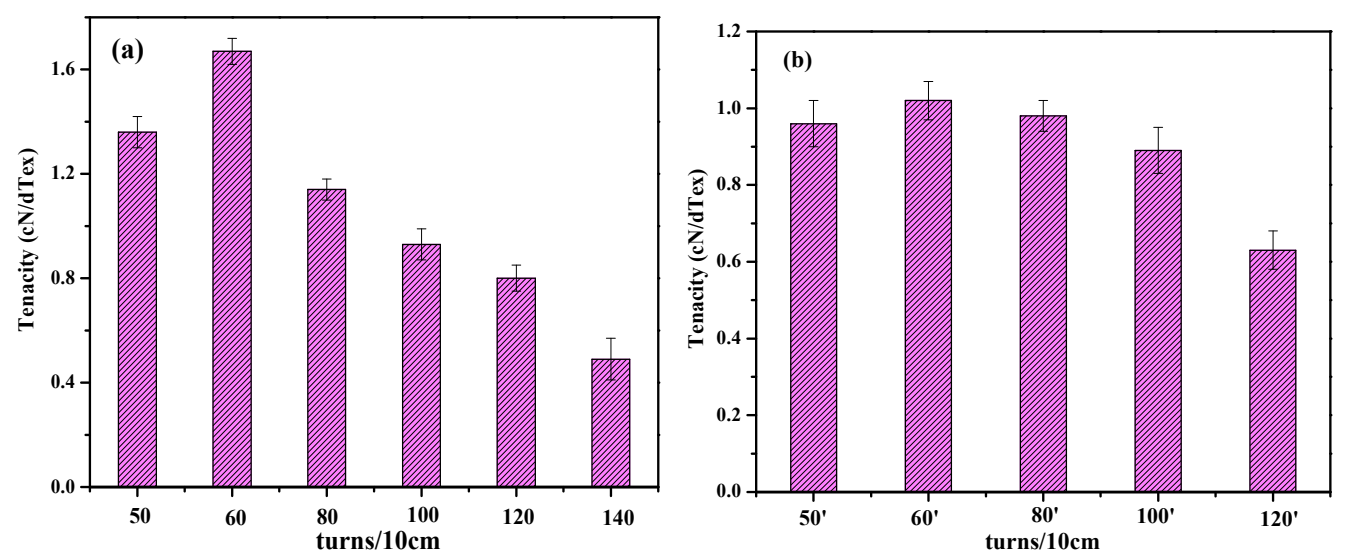

Figure 4. The breaking strength of conductive wrapped yarns as related to the twist counts. (a) one metallic wire; (b) two metallic wires.

\subsection{EMI Shielding Effectiveness of Conductive Fabrics}

The four types of fabrics all demonstrate an EMI SE that is highly dependent on whether the metallic wires and fabrics build a complete shielding network. Furthermore, the employment of superhydrophobic treatment has a positive influence on the EMI SE. When composed of a higher density, the fabrics have a smaller porosity and greater EMI SE [30,31]. After the superhydrophobic treatment, the fabrics are enwrapped in transparent films that decrease the distance among fibers to a certain extent and thus strengthens the EMI SE of the conductive fabrics. Figure 5 shows that when both the warp and weft yarns are composed of conductive wrapped yarns, the conductive fabrics exhibit a higher EMI SE comparing to those composed of conductive wrapped yarns as weft yarns. In particularly, PSA-SS-1, PSA-SS-2, PSA-SS-1' , and PSA-SS-2' have EMI SE values of 33.26, 32.50, 34.08, and $34.42 \mathrm{~dB}$ at frequencies of $0-3000 \mathrm{MHz}$ respectively, which refers to a $99 \%$ shielding effectiveness, suggesting that the conductive fabrics have a good EMI SE. Especially at $1000-2500 \mathrm{MHz}$, the fabrics have EMI SE of 30-50 dB. Furthermore, the number of metallic wires only has a marginal influence on the EMI SE because EMI SE is primarily related to a well-established shielding network.

The shielding materials that suit the majority of electronic products are required to be able to shield incident electromagnetic waves between 30 and $1000 \mathrm{MHz}$, and an EMI SE of $35 \mathrm{~dB}$ is deemed to effectively block electromagnetic waves. Accordingly, the proposed conductive fabrics are also suitable for industry and commercial electronic equipment. With PSA roving as warp yarns, the conductive fabrics have an average EMI SE lower than $10 \mathrm{~dB}$, indicating a low EMI SE. Although the difference in both of the number of laminations as well as lamination angle is helpful to the EMI SE, it also increases the thickness of the fabrics which is detrimental to the specific shielding effectiveness (SSE, the SE per unit volume). The EMI SE is based on the formation of a shielding network that is formed in the conductive fabrics. Using conductive yarns as both the warp and weft yarns can build a complete shielding network and yields a good shielding effect. If the conductive fabrics only have conductive yarns as the weft yarns, the absence of a shielding network leads to a low EMI SE. 

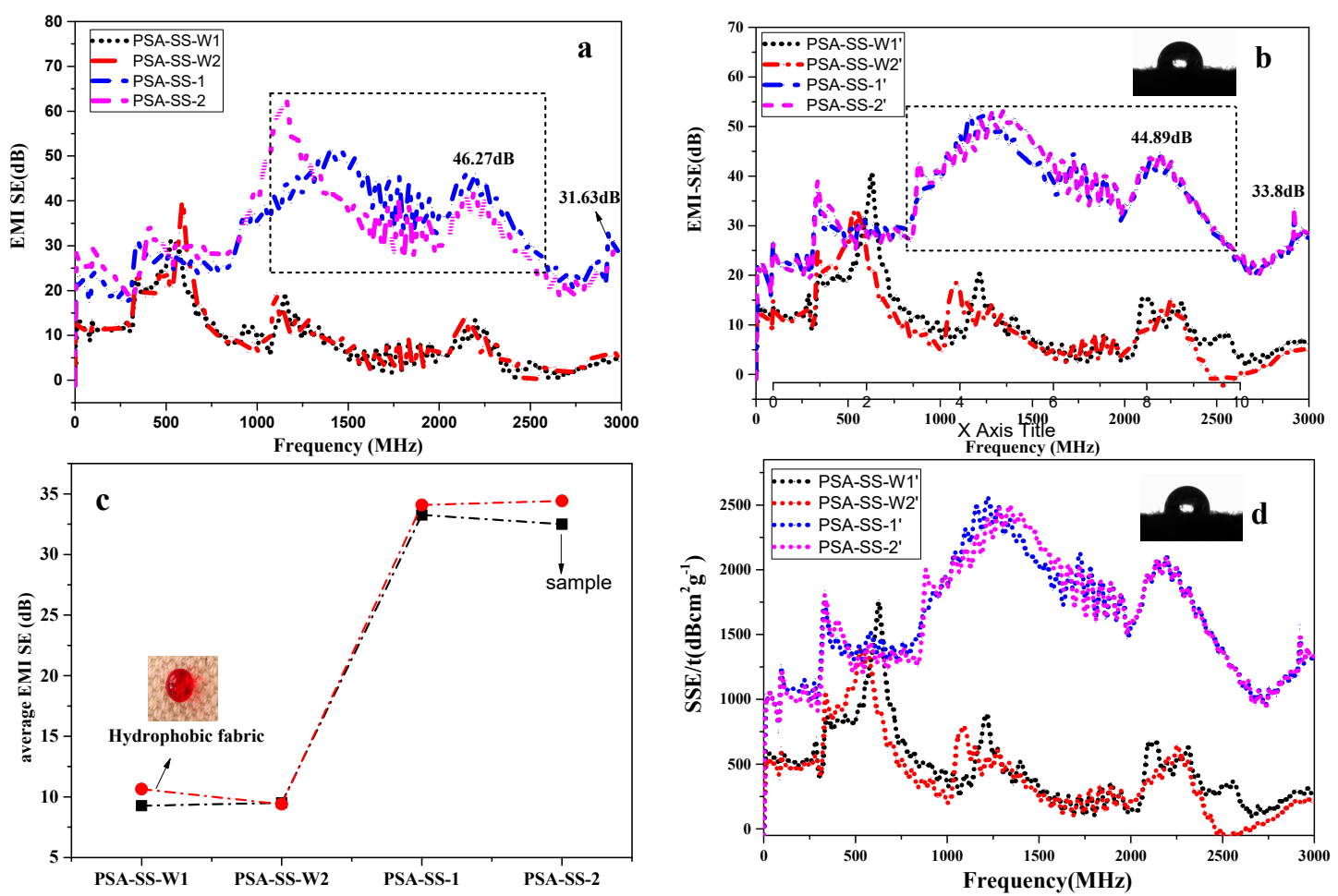

Figure 5. (a) The electromagnetic interference shielding effectiveness (EMI SE) of four fabrics without hydrophobic treatment; (b) the EMI SE of four fabrics with hydrophobic treatment; (c) The average EMI SE value of samples before and after hydrophobic treatment; (d) the shielding effectiveness per unit volume (SSE) values of hydrophobic fabrics.

To further demonstrate the highly efficient EMI shielding of the conductive composite fabrics, the shielding effectiveness per unit volume (SSE) and specific SSE/t of the fabric based EMI shielding material are discussed in Figure $5 \mathrm{~d}$ and Table 2 [31,32]. It can be found that the PSA-SS-1' and PSA-SS-2' superhydrophobic and conductive composite fabric in this study possesses a very high SSE/t $\left(1623 \mathrm{~dB} \cdot \mathrm{cm}^{2} / \mathrm{g}\right.$ and $\left.1617 \mathrm{~dB} \cdot \mathrm{cm}^{2} / \mathrm{g}\right)$. The SSE value of the conductive fabric is related to the thickness of the fabric, the density of the fabric, and the total $\mathrm{dB}$ value. The larger the SSE value, the better the shielding effect.

Table 2. The shielding effectiveness per unit volume (SSE) and SSE/t of four types of fabrics.

\begin{tabular}{|c|c|c|c|c|c|c|}
\hline Fabric Type & $\begin{array}{c}\text { Thickness } \\
\text { (mm) }\end{array}$ & $\begin{array}{l}\text { Density } \\
\left(\mathrm{g} / \mathrm{cm}^{3}\right)\end{array}$ & $\begin{array}{l}\text { EMI SE } \\
\text { (dB) }\end{array}$ & $\begin{array}{c}\mathrm{SE} / \mathrm{t} \\
(\mathrm{dB} / \mathrm{mm})\end{array}$ & $\begin{array}{c}\text { SSE } \\
\left(\mathrm{dB} \mathrm{cm}^{3} \mathrm{~g}^{-1}\right)\end{array}$ & $\begin{array}{c}\mathrm{SSE} / \mathrm{t} \\
\left(\mathrm{dB} \mathrm{cm}^{2} \mathrm{~g}^{-1}\right)\end{array}$ \\
\hline PSA-SS-W1' & 0.41 & 0.56 & 10.64 & 25.95 & 19 & 463.43 \\
\hline PSA-SS-W2' & 0.41 & 0.57 & 9.38 & 22.87 & 16.46 & 401.33 \\
\hline PSA-SS-1' & 0.38 & 0.55 & 34.08 & 89.68 & 61.96 & 1623.98 \\
\hline PSA-SS-2' & 0.38 & 0.56 & 34.42 & 90.57 & 61.46 & 1617.38 \\
\hline
\end{tabular}

\subsection{The Permittivity of Conductive Fabrics}

The permittivity' real part $\left(\varepsilon^{\prime}\right)$ represents the polarization level of the conductive fabrics in an external electric field. A high permittivity real part indicates the higher polarization ability of the material. Similarly, the permittivity's imaginary part $\left(\varepsilon^{\prime \prime}\right)$ represents the energy loss caused by the rearrangement of the electric dipole moment for the conductive fabrics in an external electric field. A high imaginary part indicates a greater loss capacity against electromagnetic waves. In addition, the loss tangent characterizes the absorption attenuation capability of the materials, and thus a greater loss tangle means an equivalently better absorbing property [33-35]. 
Figure 6 shows that PSA-SS-1 and PSA-SS-2 which are composed of conductive yarns as both warp and weft yarns have $\varepsilon^{\prime}$ and $\varepsilon^{\prime \prime}$ values that are higher than those of PSA-SS-W1 and PSA-SS-W2 which have only weft yarns that are composed of conductive yarns. PSA-SS-1 and PSA-SS-2 have a higher storage energy capacity, higher dielectric loss capability, and better EMI SE than PSA-SS-W1 and PSA-SS-W2 because of a higher polarization ability and more loss capacity. Additionally, the shielding mechanisms of EMI SE are absorption, reflection, and multiple reflections. Taking PSA-SS-1 as an example, the $\varepsilon^{\prime}$ is between 4 and 6 and the $\varepsilon^{\prime \prime}$ is between 0 and 1.2, and the latter is smaller than the former. Due to the low absorbing ability and high polarization, PSA-SS-A thus shields electromagnetic waves primarily by means of reflection.

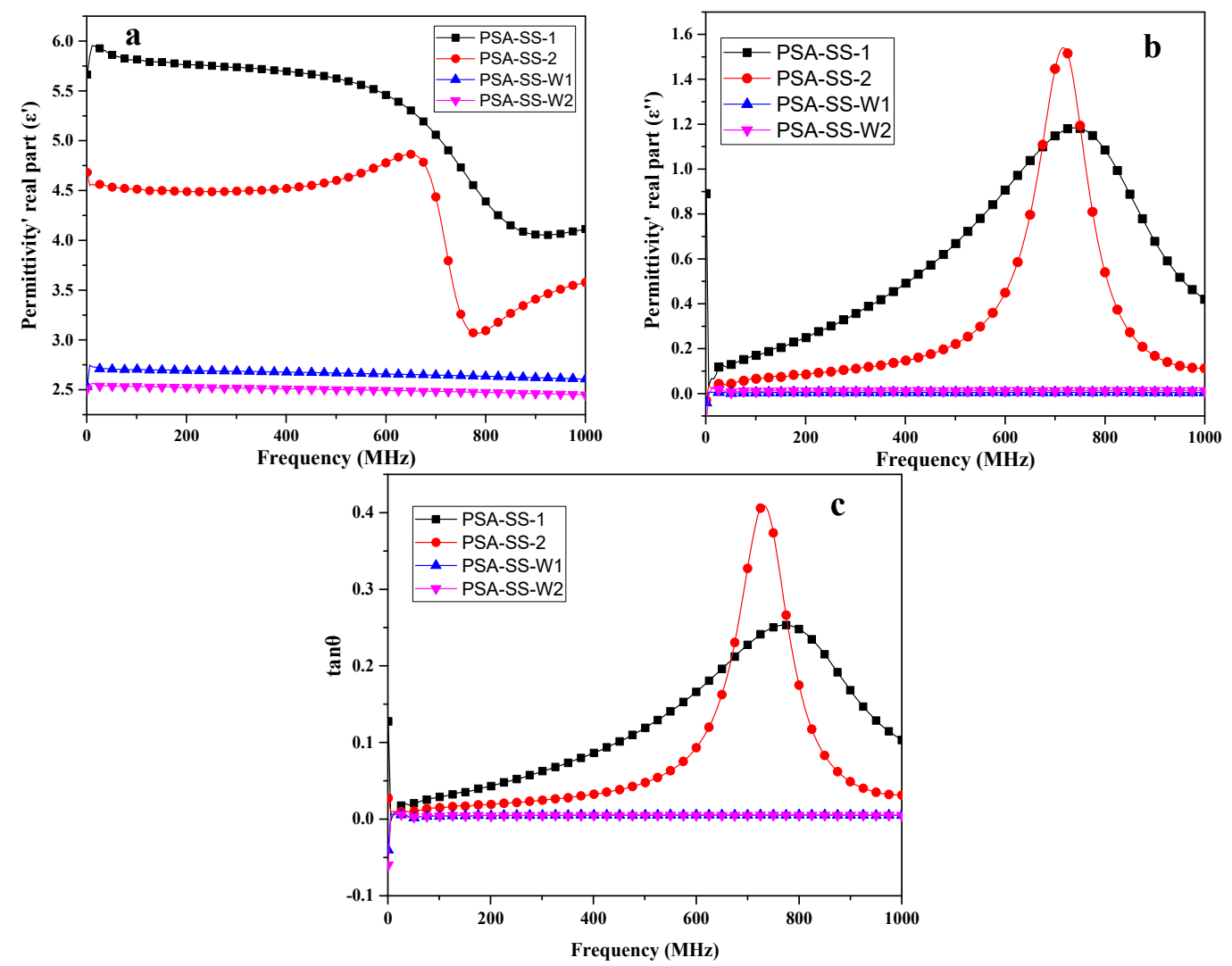

Figure 6. The influence of different wire content fabrics on the (a) permittivity's real part $\left(\varepsilon^{\prime}\right)$; (b) permittivity's imaginary part $\left(\varepsilon^{\prime \prime}\right)$ and $(\mathbf{c})$ loss tangent $(\tan \theta)$.

\subsection{The Effects of Superhydrophobic Treatment on TG, DSC and Fabric Burnt Length}

Figure 7a-c and Table 3 show that the samples before and after the hydrophobic treatment did not destroy the flame retardant property of the PSA. As for PSA-SS-W1, the thermo-gravimetric process of the PSA fiber can be divided into two stages. In the first stage, the weight loss ratio is small because it is caused by the evaporation of the residual solvent and small-molecule auxiliaries. In the second stage, the weight loss rate is high because of the decomposition of the PSA fiber. Specifically, the heat loss rate of the PSA fiber reaches the maximum at $450.8^{\circ} \mathrm{C}$, which is generally defined as its thermal decomposition temperature. With the temperature increasing to $800^{\circ} \mathrm{C}$, the residue accounts for $50.67 \%$, with half of the material not being decomposed. When it comes to the superhydrophobic-treated PSA-SS-W1', the surface is covered by waterproof $2 \mathrm{P}$ that consists of more water and small molecules. They undergo a volatile cracking process continuously with the rising temperature and thus the residue is only $29.74 \%$ at $800{ }^{\circ} \mathrm{C}$. However, PSA-SS-W1' has a thermal decomposition temperature of $454.0^{\circ} \mathrm{C}$, which is comparable to that of PSA-SS-W1, because the PSA fiber starts to decompose at this specified temperature. Usually, materials with a high thermal decomposition temperature have a better thermal 
stability. Moreover, the high residue content at the termination of the temperature indicates that there is less combustible gas. The residue has a relatively higher heat absorption, which leads to a decrease in heat energy released by the burning material. Subsequently, it is difficult to render fibers with thermal cracking, which prevents the combustion of materials and improves the combustion resistance and refractory [36-38]. Therefore, PSA roving has a good refractory and is proven to be an effective material in this study.
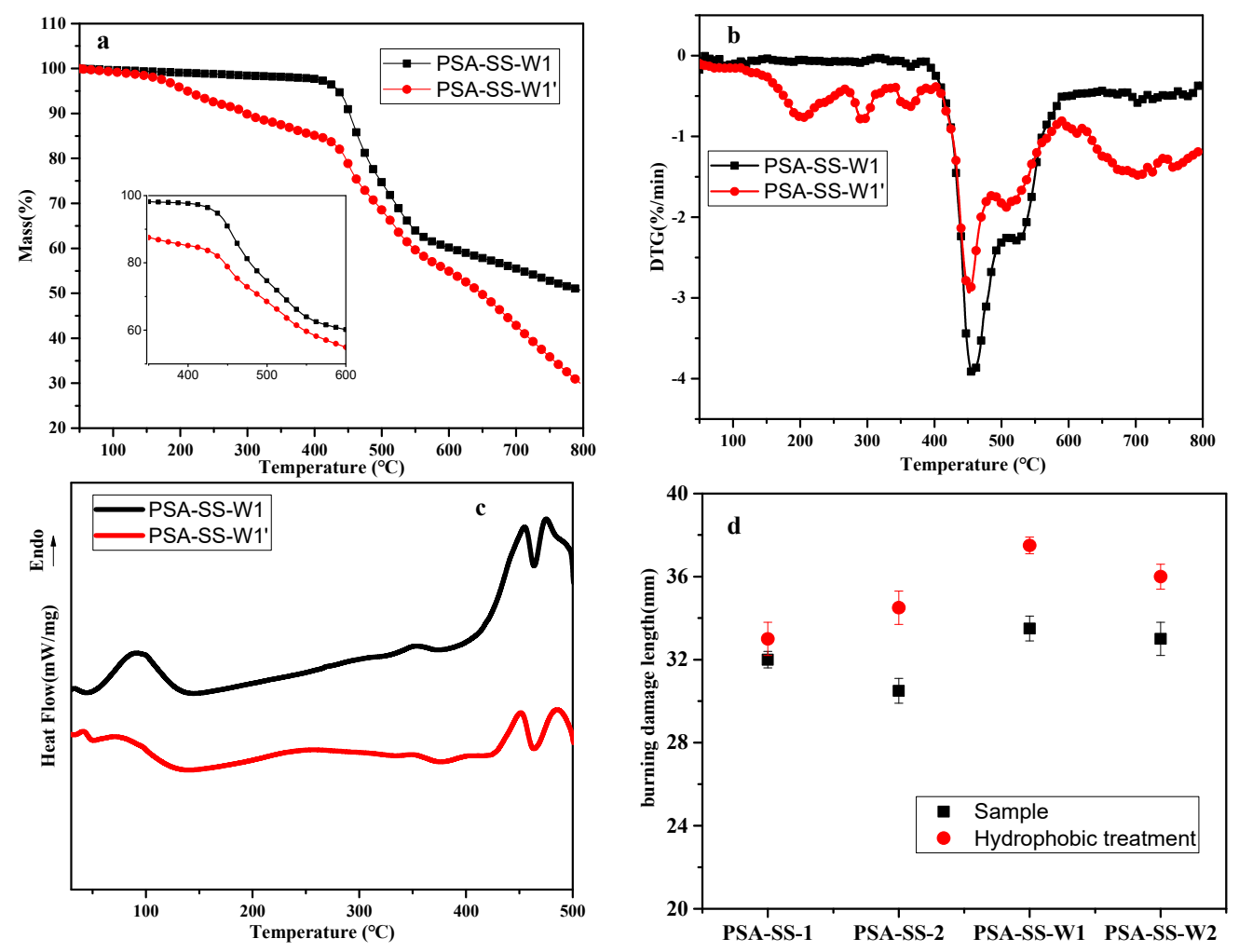

Figure 7. (a-c) The effect of hydrophobic treatment on thermal properties of fabrics; (d) the effect of hydrophobic treatment on fabric burning damage length.

Table 3. The thermogravimetric analysis (TG) and differential thermogravimetry analysis (DTG) data of fabrics under a nitrogen atmosphere as related to the employment of hydrophobic treatment.

\begin{tabular}{ccccc}
\hline Sample & $\mathbf{T}_{\mathbf{5 w t} \%}\left({ }^{\circ} \mathbf{C}\right)$ & $\mathbf{T}_{\mathbf{m a x} \mathbf{1}}\left({ }^{\circ} \mathbf{C}\right)$ & $\mathbf{T}_{\max \mathbf{2}}\left({ }^{\circ} \mathbf{C}\right)$ & Residue at $\mathbf{8 0 0}{ }^{\circ} \mathbf{C}(\mathbf{\%})$ \\
\hline PSA-SS-W1 & 435.4 & 450.8 & 519.4 & 50.67 \\
PSA-SS-W1 & 210.6 & 454.0 & 510.9 & 29.74 \\
\hline
\end{tabular}

Based on the DSC curves, the employment of superhydrophobic treatment does not affect the melting peaks. The DSC curves fluctuate significantly at $400-450{ }^{\circ} \mathrm{C}$, which means that the fibers undergo a drastic reaction equivalently. Meanwhile, no carbonized phenomenon occurred, indicating that within this temperature range, thermal cracking occurs to the fibers. Moreover, the burned length of fabrics is dependent on the density, porosity, and structure of the fabrics. To minimize the error, each sample processed with superhydrophobic treatment is used to compare the length before and after combustion. Figure $7 \mathrm{~d}$ shows that the flame-retardant property of fabrics does not compromise for the employment of superhydrophobic treatment. The burned length of PSA-SS-1, PSA-SS-2, PSA-SS-W1, and PSA-SS-W2 is $33.0 \mathrm{~mm}, 34.5 \mathrm{~mm}, 37.5 \mathrm{~mm}$, and $36 \mathrm{~mm}$, which is $1 \mathrm{~mm}, 3 \mathrm{~mm}, 4 \mathrm{~mm}$, and 3 $\mathrm{mm}$ longer than the original length, respectively. The average burned length of samples is shorter than $40 \mathrm{~mm}$. The fabrics do not demonstrate distinct shrinkage, cracking, and dripping during the combustion, after which the fabrics exhibit carbonization and have a crisp edge, retaining good fabric 
morphology. The smoldering and continuous burning times are both zero, and therefore, the thermal behaviors of fabrics are not correlated with the superhydrophobic treatment.

\section{Conclusions}

This study proposes using a specially designed feeding device to produce conductive corn yarns with PSA roving as the coated material and metallic wires as the core material. The conductive corn yarns are made into conductive fabrics and then processed with superhydrophobic treatment, forming superhydrophobic/flame retardant/electromagnetic shielding fabrics. The test results show that treated with waterproof $2 \mathrm{P}$, the fabric CAs reached $155^{\circ}$ and the EMI SE is also increased accordingly. The EMI SE value of PSA-SS-1' ${ }^{\prime}$ and PSA-SS-2' is $34.08 \mathrm{~dB}$ and $34.42 \mathrm{~dB}$ with electromagnetic waves of 0-3000 MHz. Comparing to the control groups, the EMI SE value of PSA-SS- $1^{\prime}$ and PSA-SS-2' is $0.82 \mathrm{~dB}$ and $1.92 \mathrm{~dB}$ higher. The shielding effectiveness of $99.9 \%$ proves that the conductive fabrics are suitable for use in harsh conditions such as humid, acid and alkali environments. Based on the permittivity results, when composed of conductive yarns for both the warp and weft yarns, the conductive fabrics have a polarization ability and greater loss ability against electromagnetic waves than those made of only weft yarns being conductive yarns in an external electric field. The fabrics shield electromagnetic waves by means of reflection. After the hydrophobic treatment with waterproof $2 \mathrm{P}$, the PSA fiber retains its inherent thermal properties demonstrated by a burned length of the conductive fabrics being shorter than $40 \mathrm{~mm}$.

Author Contributions: In this study, the concepts and designs for the experiment are supervised by J.-H.L. and H.-K.P.; Experiment and data processing are conducted by Y.W.; Text composition and results analysis are performed by H.-K.P. and Y.W.; The experimental result is examined by T.-T.L., C.-W.L. and Q.H.

Acknowledgments: This work was supported by National Natural Science Foundation of China [grant numbers 51503145, 21806121, 11702187]; the Natural Science Foundation of Tianjin City [grant numbers 18JCQNJC03400, 17JCQNJC08000]; the Natural Science Foundation of Fujian Province [grant numbers 2018J01504, 2018J01505]; the Opening Project of Green Dyeing and Finishing Engineering Research Center of Fujian University (2017001A, 2017001B, 2017002B and 2017004B), and the Program for Innovative Research Team in University of Tianjin [grant number TD13-5043].

Conflicts of Interest: The authors declare no conflict of interest.

\section{References}

1. Shahzad, F.; Alhabeb, M.; Hatter, C.B.; Anasori, B.; Man Hong, S.; Koo, C.M. Electromagnetic interference shielding with $2 \mathrm{~d}$ transition metal carbides (mxenes). Science 2016, 353, 1137-1140. [CrossRef] [PubMed]

2. Eddib, A.A.; Chung, D.D.L. The importance of the electrical contact between specimen and testing fixture in evaluating the electromagnetic interference shielding effectiveness of carbon materials. Carbon 2017, 117, 427-436. [CrossRef]

3. Huang, C.L.; Lou, C.W.; Liu, C.F.; Huang, C.H.; Song, X.M.; Lin, J.H. Polypropylene/graphene and polypropylene/carbon fiber conductive composites\&58; mechanical, crystallization and electromagnetic properties. Appl. Sci. 2015, 5, 1196-1210.

4. Drakakis, E.; Kymakis, E.; Tzagkarakis, G.; Louloudakis, D.; Katharakis, M.; Kenanakis, G.; Suchea, M.; Tudose, V.; Koudoumas, E. A study of the electromagnetic shielding mechanisms in the GHz frequency range of graphene based composite layers. Appl. Surf. Sci. 2017, 398, 15-18. [CrossRef]

5. Tana, Y.J.; Lia, J.; Gao, Y.; Li, J.; Guo, S.Y.; Wang, M. A facile approach to fabricating silver-coated cotton fiber non-woven fabrics for ultrahigh electromagnetic interference shielding. Appl. Surf. Sci. 2018, 458, 236-244. [CrossRef]

6. Lin, S.C.; Ma, C.C.M.; Hsiao, S.T.; Wang, Y.S.; Yang, C.Y.; Liao, W.H. Electromagnetic interference shielding performance of waterborne polyurethane composites filled with silver nanoparticles deposited on functionalized graphene. Appl. Surf. Sci. 2016, 385, 436-444. [CrossRef]

7. Jung, J.; Lee, H.; Ha, I.; Cho, H.; Kim, K.K.; Kwon, J. Highly Stretchable and Transparent Electromagnetic Interference Shielding Film Based on Silver Nanowire Percolation Network for Wearable Electronics Applications. ACS Appl. Mater. Interfaces 2017, 9, 44609-44616. [CrossRef] 
8. Thomassin, J.M.; Jérôme, C.; Pardoen, T. Polymer/carbon based composites as electromagnetic interference (EMI) shielding materials. Mater. Sci. Eng. R 2013, 74, 211-232. [CrossRef]

9. Cao, W.T.; Chen, F.F.; Zhu, Y.J. Binary Strengthening and Toughening of MXene/Cellulose Nanofiber Composite Paper with Nacre-Inspired Structure and Superior Electromagnetic Interference Shielding Properties. ACS Nano 2018, 12, 4583-4593. [CrossRef] [PubMed]

10. Park, S.H.; Theilmann, P.T.; Asbeck, P.M.; Bandaru, P.R. Enhanced Electromagnetic Interference Shielding Through the Use of Functionalized Carbon-Nanotube-Reactive Polymer Composites. IEEE Trans. Nanotechnol. 2010, 9, 464-469. [CrossRef]

11. Lee, T.W.; Lee, S.E.; Jeong, Y.G. Carbon nanotube/cellulose papers with high performance in electric heating and electromagnetic interference shielding. Compos. Sci. Technol. 2016, 131, 77-87. [CrossRef]

12. Cheng, K.B.; Lee, M.L.; Ramakrishna, S. Electromagnetic Shielding Effectiveness of Stainless Steel/Polyester Woven Fabrics. Text. Res. J. 2001, 71, 42-49. [CrossRef]

13. Roh, J.S.; Chi, Y.S.; Kang, T.J. Electromagnetic Shielding Effectiveness of Multifunctional Metal Composite Fabrics. Text. Res. J. 2008, 78, 825-835. [CrossRef]

14. Erdumlu, N.; Saricam, C. Electromagnetic shielding effectiveness of woven fabrics containing cotton/metal-wrapped hybrid yarns. J. Ind. Text. 2016, 46, 1084-1103. [CrossRef]

15. Lou, C.W.; Lin, T.A.; Chen, A.P. Stainless steel/polyester woven fabrics and copper/polyester woven fabrics: Manufacturing techniques and electromagnetic shielding effectiveness. J. Ind. Text. 2015, 46, 214-236. [CrossRef]

16. Chen, H.C.; Lee, K.C.; Lin, J.H.; Koch, M. Fabrication of conductive woven fabric and analysis of electromagnetic shielding via measurement and empirical equation. J. Mater. Process. Technol. 2007, 1, 124-130. [CrossRef]

17. Lin, A.D.; Kung, C.L.; Hsieh, W.C.; Hsu, C.M.; Chen, C.Y. Study on cleaning the surface of stainless steel 316 using plasma electrolysis technology. Appl. Sci. 2018, 8, 1060. [CrossRef]

18. Huang, X.; Dai, B.; Ren, Y.; Xu, J.; Zhu, P. Preparation and Study of Electromagnetic Interference Shielding Materials Comprised of Ni-Co Coated on Web-Like Biocarbon Nanofibers via Electroless Deposition. J. Nanomater. 2015, 2015, 1-7. [CrossRef]

19. Shen, B.; Zhai, W.; Tao, M.; Ling, J.; Zheng, W. Lightweight, Multifunctional Polyetherimide/Graphene@ $\mathrm{Fe}_{3} \mathrm{O}_{4}$ Composite Foams for Shielding of Electromagnetic Pollution. ACS Appl. Mater. Interfaces 2013, 5, 11383-11391. [CrossRef]

20. Zou, L.; Zhang, S.; Li, X.; Lan, C.; Qiu, Y.; Ying, M. Nanocomposites: Step-by-step strategy for constructing multilayer structured coatings toward high-efficiency electromagnetic interference shielding. Adv. Mater. Interfaces 2016, 3, 1-6.

21. Xing, Y.; Xue, Y.; Song, J.; Sun, Y.; Huang, L.; Liu, X.; Sun, J. Superhydrophobic coatings on wood substrate for self-cleaning and EMI shielding. Appl. Surf. Sci. 2018, 436, 865-872. [CrossRef]

22. Sui, Y.W.; Sun, Z.; Wang, X.H. Microstructure and friction performance of copper film fabricated by ion implantation assisted electroless plating on PTFE. Mater. Sci. Technol. 2011, 27, 1680-1685. [CrossRef]

23. Chen, H.C.; Lee, K.C.; Lin, J.H.; Koch, M. Comparison of electromagnetic shielding effectiveness properties of diverse conductive textiles via various measurement techniques. J. Mater. Process. Technol. 2007, 192-193, 549-554. [CrossRef]

24. Wu, M.; Li, Y.; An, N.; Sun, J. Applied Voltage and Near-Infrared Light Enable Healing of Superhydrophobicity Loss Caused by Severe Scratches in Conductive Superhydrophobic Films. Adv. Funct. Mater. 2016, 26, 6777-6784. [CrossRef]

25. Rung, S.; Schwarz, S.; Götzendorfer, B.; Esen, C.; Hellmann, R. Time dependence of wetting behavior upon applying hierarchic nano-micro periodic surface structures on brass using ultra short laser pulses. Appl. Sci. 2018, 8, 700. [CrossRef]

26. Baliga, S.; Wong, W.T. Depolymerization of poly(ethylene terephthalate) recycled from post-consumer soft-drink bottles. J. Polym. Sci. Polym. Chem. 1989, 27, 2071-2082. [CrossRef]

27. Kandola, B.K.; Horrocks, A.R.; Myler, P.; Blair, D. Mechanical performance of heat/fire damaged novel flame retardant glass-reinforced epoxy composites. Compos. Part A-Appl. Sci. Manuf. 2003, 34, 863-873. [CrossRef]

28. Araby, S.; Wang, C.H.; Wu, H.; Meng, Q.; Kuan, H.C.; Kim, N.K. Development of flame-retarding elastomeric composites with high mechanical performance. Compos. Part A-Appl. Sci. Manuf. 2018, 109, 257-266. [CrossRef] 
29. Jiarong, R. Thermal treated analysis of PSA fiber. Melliand China 2007, 1, 27-33.

30. Han, J.; Wang, X.; Qiu, Y.; Zhu, J.; Hu, P.A. Infrared-transparent films based on conductive graphenenetwork fabrics for electromagnetic shielding. Carbon 2015, 87, 206-214. [CrossRef]

31. Xing, D.; Lu, L.; Teh, K.S.; Wan, Z.; Xie, Y.; Tang, Y. Highly flexible and ultra-thin Ni-plated carbon-fabric/polycarbonate film for enhanced electromagnetic interference shielding. Carbon 2018, 132, 32-41. [CrossRef]

32. Chen, Z.; Yi, D.; Shen, B.; Zhang, L.; Ma, X.; Pang, Y.; Liu, L.; Wei, X.; Zheng, W. Semi-transparent biomass-derived macroscopic carbon grids for efficient and tunable electromagnetic shielding. Carbon 2018, 139, 271-278. [CrossRef]

33. Liu, Y.; Zhao, X.; Xiao, T. Preparation of polypyrrole coated cotton conductive fabrics. J. Text. Inst. 2016, 108, 829-834. [CrossRef]

34. Liu, Y.; Liu, Y.; Zhao, X. The research of EM wave absorbing properties of ferrite/silicon carbide double coated polyester woven fabric. J. Text. Inst. 2017, 109, 1-7. [CrossRef]

35. Arjmand, M.; Apperley, T.; Okoniewski, M.; Sundararaj, U. Comparative study ofelectromagnetic interference shielding properties of injection molded versus compression molded multi-walled carbon nanotube/polystyrene composites. Carbon 2012, 50, 5126-5134. [CrossRef]

36. Al-Saleh, M.H.; Saadeh, W.H.; Sundararaj, U. EMI shielding effectiveness of carbon based nanostructured polymeric materials: A comparative study. Carbon 2013, 60, 146-156. [CrossRef]

37. Jia, X.; Li, G.; Yu, Y.; Sui, G.; Yang, X. Ablation and thermal properties of ethylene-propylene-diene elastomer composites reinforced with polysulfonamide short fibers. J. Appl.Polym. Sci. 2009, 113, 283-289. [CrossRef]

38. Soulier, J.; Chabert, B.; Chauchard, J. Synthesis and properties of some polyamides and polysulfonamides. J. Appl. Polym. Sci. 2010, 18, 2435-2447. [CrossRef]

(C) 2019 by the authors. Licensee MDPI, Basel, Switzerland. This article is an open access article distributed under the terms and conditions of the Creative Commons Attribution (CC BY) license (http://creativecommons.org/licenses/by/4.0/). 